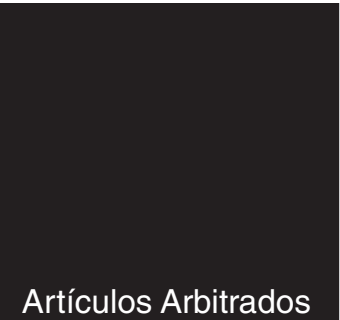

\title{
Ciudad dispersa y fragmentada Lecturas de forma urbana en emprendimientos habitacionales privados, Córdoba 2001-2010
}

\section{Cecilia Marengo}

Arquitecta, magíster en Diseño Arquitectónico y Urbano, Universidad Nacional de Córdoba Argentina. Ph.D Universidad Tecnológica de Delft, Holanda, Departamento de Renovación Urbana y Vivienda. Investigadora independiente del Consejo Nacional de Investigaciones Científicas y Técnicas (CONICET). Profesora titular FAUD-UNC. Subdirectora del Instituto de Investigación de Vivienda y Hábitat (INVIHAB-FAUD UNC). mcmarengo@gmail.com.

\section{Martín Lemma}

Arquitecto (FAUD-UNC). Becario interno doctoral (CONICET) con sede en el Instituto de Investigación de Hábitat y Vivienda (INVIHAB-UNC). Doctorando en Estudios Urbanos (UNGS), Argentina.

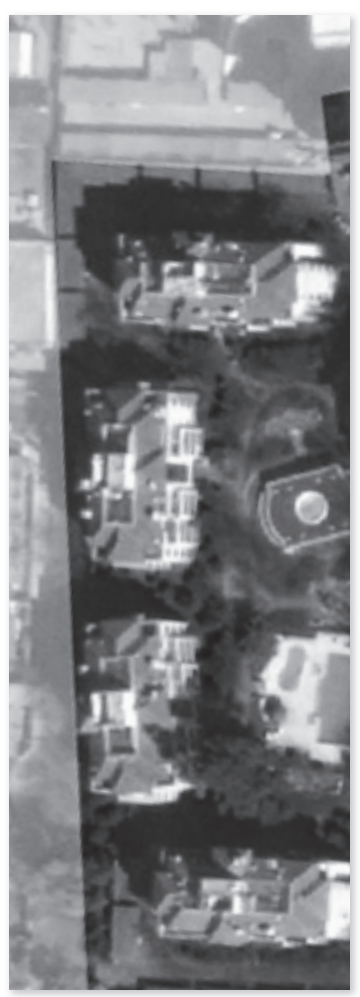




\section{Resumen}

En las últimas décadas del siglo XX, las ciudades latinoamericanas han experimentado cambios significativos, como consecuencia de los procesos de globalización a escala mundial que han afectado los patrones de crecimiento, no solo a escala regional, sino en la dimensión espacial intraurbana. El presente artículo examina los desarrollos residenciales construidos por el mercado inmobiliario en la ciudad de Córdoba, Argentina (período 2001-2010), da cuenta de las transformaciones en las nuevas expansiones periféricas (integradas por urbanizaciones residenciales especiales URE) e incorpora en el análisis nuevas formas urbanas localizadas en sectores consolidados de la ciudad (complejos de casonas).

La lectura de estos tipos residenciales aporta indicadores (dimensiones, densidad y distancias al centro), permite caracterizar las intervenciones y entender su evolución en el tiempo. La investigación de las formas resultantes, en el espacio local donde se presentan, facilitará formular modelos interpretativos para planificar la ciudad, de cara a los desafíos del siglo XXI.

\section{Palabras clave}

Forma urbana; emprendimientos residenciales privados; Municipio de Córdoba.

\section{Abstract}

Dispersed and Fragmented city. observations on urban form in private developments. Cordoba (2001-2010) In the last decades of the twentieth century, Latin American cities have experienced significant changes because of globalization processes that have affected growth patterns, not only at regional scale but also at the intra-urban spatial dimension.

The present article examines the residential developments built through the real estate market in the City of Córdoba, Argentina, (during the 2001-2010 period), by reporting on the transformations in the new peripheral expansions (integrated by special residential developments: URE) and incorporating in the analysis new urban forms located in consolidated sectors of the city (housing complexes: casonas).

The analysis of these residential types, provides indicators (dimensions, density and distances to the town center), to characterize these interventions and to understand their evolution over time. Research on the resulting forms, in their local spaces, will allow the formulation of interpretive models for future city planning, facing the challenges of the 21st century.

\section{Keywords}

Urban form; private residential developments; City of Córdoba. 


\section{Transformaciones urbanas y hábitat residencial}

Desde las últimas décadas del siglo XX, las ciudades latinoamericanas han experimentado cambios significativos en las relaciones de producción del capitalismo, como consecuencia de los procesos de globalización a escala mundial. La geografía de la globalización se caracteriza por la autonomía de los flujos con respecto a los lugares y la creciente deslocalización de las empresas hacia emplazamientos periféricos que ofrecen mayor rentabilidad, posibilitada por los avances tecnológicos y de comunicación (CASTELLS, 1995; MARcuse \& VAN KemPen, 2000; SASSEN, 2002). Numerosos estudios analizan los efectos de la relocalización de actividades económicas y la reestructuración del mercado laboral, tanto en los sistemas urbanos de ciudades desarrolladas como en vías de desarrollo, identificando los efectos de las transformaciones económicas en el espacio urbano.

Los cambios significativos en la vida urbana, resultantes de los procesos antes señalados, han afectado las condiciones económicas, sociales, culturales y los patrones físico-espaciales de crecimiento en las aglomeraciones no solo a escala regional, sino también en la dimensión espacial intra-urbana (De MATTOs, 2010; Jenks, KozAK, \& TAKKANON, 2008). A escala regional, se menciona la conformación de regiones policéntricas, como consecuencia de la descentralización de funciones y equipamientos, las mejoras en las infraestructuras de movilidad metropolitana y la migración de la población. ${ }^{1}$ A escala urbana, si bien inciden estos mismos procesos, el acento está puesto en la fragmentación del tejido residencial, tanto en los emprendimientos residenciales cerrados localizados en las nuevas periferias (débilmente conectados, dependientes del automóvil y con patrones de ocupación del suelo diferentes de los desplegados en la etapa de industrialización), como en áreas en proceso de renovación.

Los cambios en los modos de habitar se traducen en nuevas formas residenciales promovidas por iniciativa privada, que dan respuesta a las demandas de seguridad, acceso al espacio exterior, servicios o equipamientos protegidos. Estas modifican sustancialmente las formas del tejido residencial presentes en las etapas precedentes por, al menos, dos rasgos característicos: la declinación en las densidades edilicias y la escala de las operaciones inmobiliarias. ${ }^{2}$ En áreas consolidadas, asociadas a procesos de renovación urbana, grandes proyectos urbanos re-significan e intensifican usos y aprovechan las ventajas de localizaciones vacantes u obsoletas por la migración de funciones industriales, o de servicios, hacia la periferia.
1. DE MATTOS (2001) analiza las transformaciones urbanas en la región latinoamericana asociadas con el proceso de reestructuración económica. Señala que el crecimiento de la mancha urbana se encamina a un proceso de metropolización expandida, que va integrando pueblos, áreas rurales y desbordando los límites de la urbanización, en una dinámica de alcance regional, de estructuras urbanas policéntricas y fronteras difusas.

2. SHLOMо ет ÁL. (2010) confirman como rasgo característico de la urbanización actual la evolución de los patrones de uso del suelo hacia un modelo de ocupación disperso, que se caracteriza por la disminución de las densidades edilicias brutas en las ciudades y un proceso de ocupación de suelo discontinuo y fragmentado. 


\section{Cecilia Marengo y Martín Lemma}

3. Ciudad difusa se aborda como forma no morfológica, sino de organización espacial del territorio del Véneto una región en el nordeste de Italia que tiene como capital a la ciudad de Venecia. Se presenta como superadora del concepto de urbanización difusa, e indica diferentes estadios en el proceso de organización de un territorio.
Indovina (2004) propone el concepto de "ciudad difusa” como evolución de lo que se consideraba como la urbanización difusa, para designar un estadio diferente en la organización de un territorio urbano en expansión, que se produce como consecuencia de la reorganización de los procesos económicos. ${ }^{3}$ Constata que la importancia de las transformaciones llevaría a identificar una nueva fase que difiere de la mera continuidad de los fenómenos preexistentes, o de suburbanización con la migración de residencias hacia la periferia. Inicialmente, la urbanización difusa se caracterizó por la localización de residencias dispersas dentro de los límites del propio municipio, ausencia de servicios y una estructura de asentamiento en islas, con conexiones poco relevantes entre ellas. Una segunda fase corresponde a una migración residencial por insatisfacción con la ciudad consolidada, en la que se identifican dos tipos de situaciones: una asociada a los parámetros de oferta y costo de las viviendas, en la cual el precio sería el factor clave que facilitaría localizaciones más accesibles (en términos de oferta residencial), lo que ha contribuido al crecimiento metropolitano. En este sentido, se mencionan los cambios derivados de las preferencias de sectores poblacionales de alto ingreso, que eligen localizarse en nuevas urbanizaciones dispersas promovidas por el mercado fortaleciendo un modelo de residencia en baja densidad, en un entorno natural, dependiente de movilidad privada en auto.

Para INDOVINA (2004: 52), "el modo de relacionarse de los individuos y familias con la ciudad no constituye ni una constante ni una determinación 'natural', sino que más bien es un producto cultural y político". Es decir que la idea misma de ciudad como espacio que concentra densidad (poblacional y edilicia) e intensidad de usos puede ser reemplazada por la idea de ciudad difusa en tanto espacio donde las condiciones de accesibilidad y movilidad median entre la distancia que impone esta forma de ocupación. El concepto de habitar, con una connotación colectiva de la ciudad, de acceso a bienes y servicios urbanos (en un modelo de ciudad concentrada) estaría modificándose en el caso de la ciudad difusa, donde la movilidad y la accesibilidad (en términos de tiempos de desplazamiento) estarían reemplazando las condiciones de alta densidad y de intensidad (propias de la ciudad consolidada), en tanto posibilidad para una población numerosa de acceder fácilmente a cada punto del territorio.

En este contexto, no se puede abordar el estudio de los nuevos modelos residenciales sin considerar que las transformaciones urbanas recientes están sujetas a las relaciones sociales de producción y a la lógica capitalista. Como menciona DE MATTos (2010: 172), en las 
presentes condiciones regidas por relaciones sociales capitalistas tienden a fortalecerse tres aspectos fundamentales: la posibilidad de alcanzar mayores niveles de inversión y crecimiento económico, que se encuentra condicionada por la ganancia que esperan obtener los propietarios o administradores del capital; con la formación y expansión de un espacio capitalista de acumulación de cobertura planetaria, las perspectivas de crecimiento en cada uno de esos lugares están cada día más férreamente regidas por las condiciones que allí se ofrezcan para la valorización privada y, en tercer lugar, la factibilidad de promover objetivos sociales de interés general (en tanto principios del urbanismo) está condicionada por la compatibilidad de esos objetivos con los de los propietarios del capital.

En el presente artículo nos proponemos examinar los desarrollos residenciales construidos por el mercado inmobiliario en la ciudad de Córdoba, Argentina, en la primera mitad del siglo XXI, dando cuenta de las transformaciones del espacio residencial de producción privada presentes en las nuevas expansiones periféricas en el período 2001-2010 (integradas por urbanizaciones residenciales especiales URE), como también incorporar en el análisis las nuevas formas urbanas localizadas en sectores consolidados de la ciudad (designados como complejos de casonas).

El artículo aborda el estudio de estos dos tipos urbanos residenciales aportando indicadores sobre su evolución en el período (dimensiones, densidad y distancias al centro), considerando, como menciona Neuman (2005), que la forma es tanto el parámetro que permite caracterizar las intervenciones, describirlas, como entender su evolución en el tiempo. El concepto de ciudad difusa (InDovina, 2004) remite a la forma de organización espacial del territorio, no solo a su representación. En ambas interpretaciones de los autores antes señalados, se rescata la idea de forma que condensa, en un resultado final, los procesos que dieron lugar a su producción. En este sentido, se entiende por forma urbana a los sistemas de relaciones y localizaciones de los elementos tangibles de la ciudad en sus diferentes escalas, comprendiendo que son el resultado de la acumulación e integración - a lo largo del tiempo- de acciones regidas por tradiciones culturales, que se formalizan por fuerzas socioeconómicas (Moudon, 1997). Más aún, se asume que los proyectos urbanísticos que en este artículo se analizan son parte de los elementos estructurantes de la forma urbana y son, a su vez, el resultado de diversas maneras de combinar las operaciones de parcelación, urbanización y edificación en el tiempo y el espacio (De SolÀ-MoralEs, 1973). Entonces, el crecimiento urbano responde a un momento específico de la producción de la ciudad, y las formas del 
crecimiento urbano se piensan a partir de la concreción de los procesos de crecimiento, de la clasificación de la formación histórica de la ciudad y de la expresión de distintos modos de gestión (en este caso, privada).

La necesidad de atraer inversiones a la ciudad lleva a modificar las regulaciones urbanísticas vigentes para responder a las demandas de los agentes y establecer nuevas condiciones para dar lugar al desarrollo de proyectos residenciales que promueve la inversión privada. En este contexto, nos preguntamos:

-¿Qué lecciones podemos extraer al examinar las formas construidas, en los nuevos emprendimientos residenciales promovidos por el mercado inmobiliario en la ciudad de Córdoba?

-¿Qué reflexiones derivan de su materialización en tanto modelos dominantes que impulsa por el mercado inmobiliario?

-¿Cuál es el modelo de ciudad que el mercado contribuye a consolidar?

La investigación y análisis críticos de las formas urbanas resultantes de estos procesos, en el espacio local donde se presentan, permitirá formular modelos interpretativos desde los cuales planificar la ciudad, de cara a los desafíos del siglo XXI.

La metodología de análisis se basó en el registro y relevamiento de todos los casos materializados en la ciudad a partir de datos aportados por la Dirección de Planeamiento de la Municipalidad de Córdoba, por empresas desarrollistas y por las herramientas de medición de fotografías digitales de Google Earth. Se consideró importante consignar las dimensiones del predio y la cantidad de unidades de vivienda, para estimar los valores de densidad bruta en las diferentes propuestas habitacionales. Si bien interesa el período 2001-2010 a los efectos de registrar la evolución de ambas tipologías, se incorporaron datos de los emprendimientos construidos en la última década del siglo XX, para el caso de las URE, y de la segunda década del siglo XXI, para casonas. Las diferentes urbanizaciones se localizaron en la estructura urbana utilizando el programa QGIS. Finalmente, se calcularon las distancias (en tiempo) en medio de transporte público y en auto particular con el programa Google Maps, para construir un dato comparativo de accesibilidad en los diferentes casos que integran la muestra de estudio. 
Emprendimientos habitacionales promovidos por el mercado inmobiliario para sectores de altos ingresos

En la última década del siglo XX se inicia en la ciudad de Córdoba un proceso de extensión de la planta urbana impulsado por los nuevos desarrollos residenciales que promueve el mercado inmobiliario para segmentos de altos-muy altos ingresos, a través de urbanizaciones residenciales especiales (URE). En la primera década del nuevo siglo, continúa esa tendencia y se presenta además un nuevo tipo de urbanización (también promovida por las empresas desarrollistas del medio) que se localiza en tejidos urbanos consolidados, aprovechando predios vacantes o con obsolescencia edilicia o de uso, que promueve una urbanización colectiva en bloques de media altura denominados "casonas". La tipología constituye una oferta innovadora en el medio local, dado que ofrece una forma de organización intermedia entre la vivienda individual aislada en lote de uso propio y la torre de departamentos (exenta o entre medianeras) en propiedad horizontal.

Ambos tipos de formas residenciales se presentan como innovadores respecto de lo que el mercado venía desarrollando en la ciudad (casa en barrio jardín en los suburbios, casas con patio en barrios consolidados o edificios de vivienda en altura) y responden a las demandas de seguridad conformando barrios cerrados y privatización de los espacios exteriores comunes del conjunto para el uso común de los residentes (con homogeneidad social) (figura 1).

Tanto las URE como las casonas representan, además, casos paradigmáticos de fragmentación urbana. Este término, si bien polisémico en el análisis urbano, señala una forma física que muestra materialización en sus límites, discontinuidad en la red de infraestructuras de movilidad/accesibilidad y carencia de espacios exteriores públicos. En el caso de las urbanizaciones residenciales especiales, impacta la escala de las intervenciones (no solo porque los predios presentan amplias superficies que abarcan desde las 172 a las 5,9 hectáreas), sino, además, porque estas se localizan de manera contigua en la estructura urbana. Con respecto a las casonas, si bien en ellas la escala de las intervenciones es menor (y las dimensiones de los predios están comprendidas entre las 2,3 a las 0,2 hectáreas de superficie), se constituyen en enclaves cerrados insertos en el tejido urbano consolidado, del que se diferencian por las formas de parcelación de lote, el emplazamiento de los edificios y los criterios de ocupación y uso del espacio. 

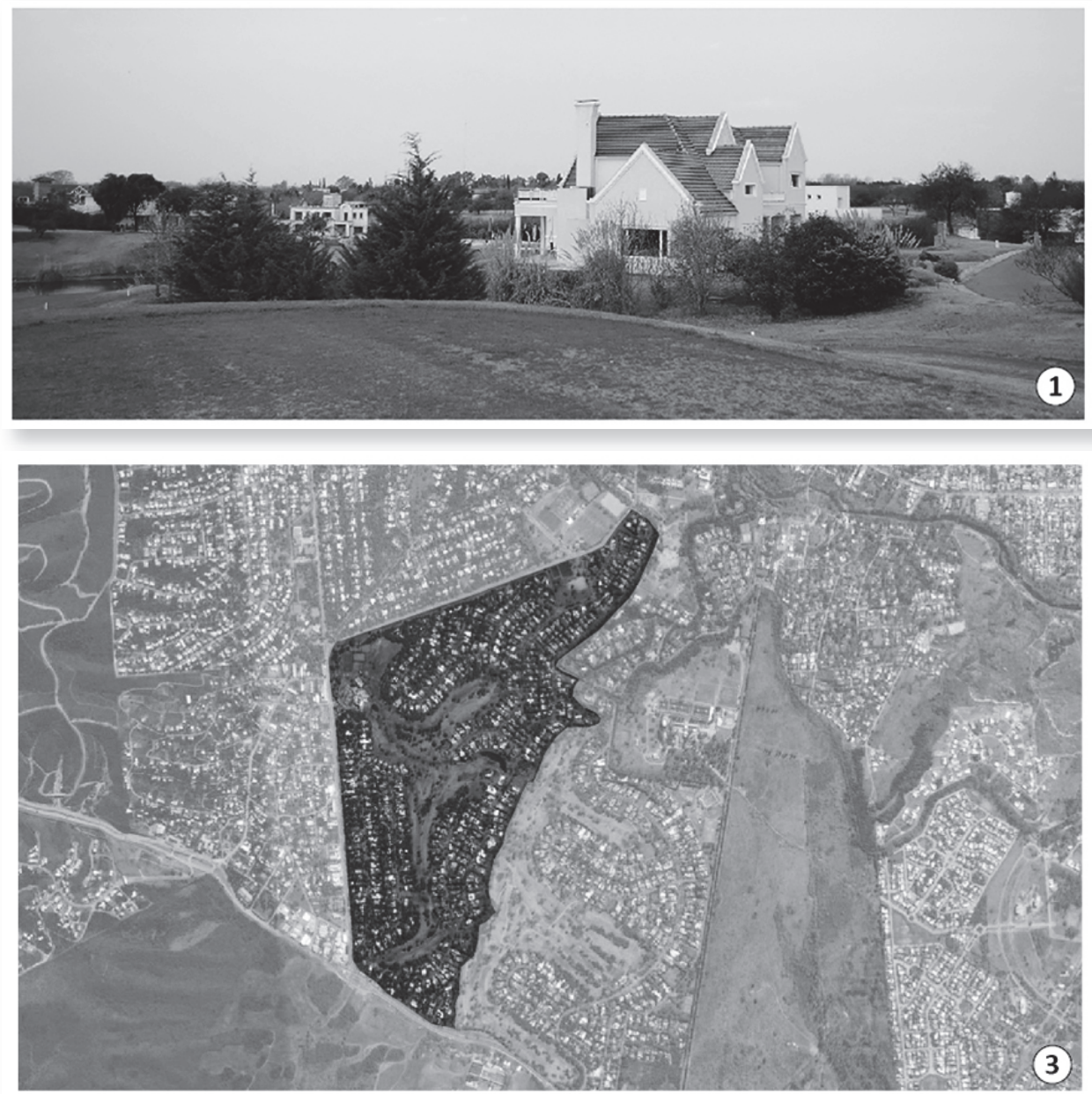

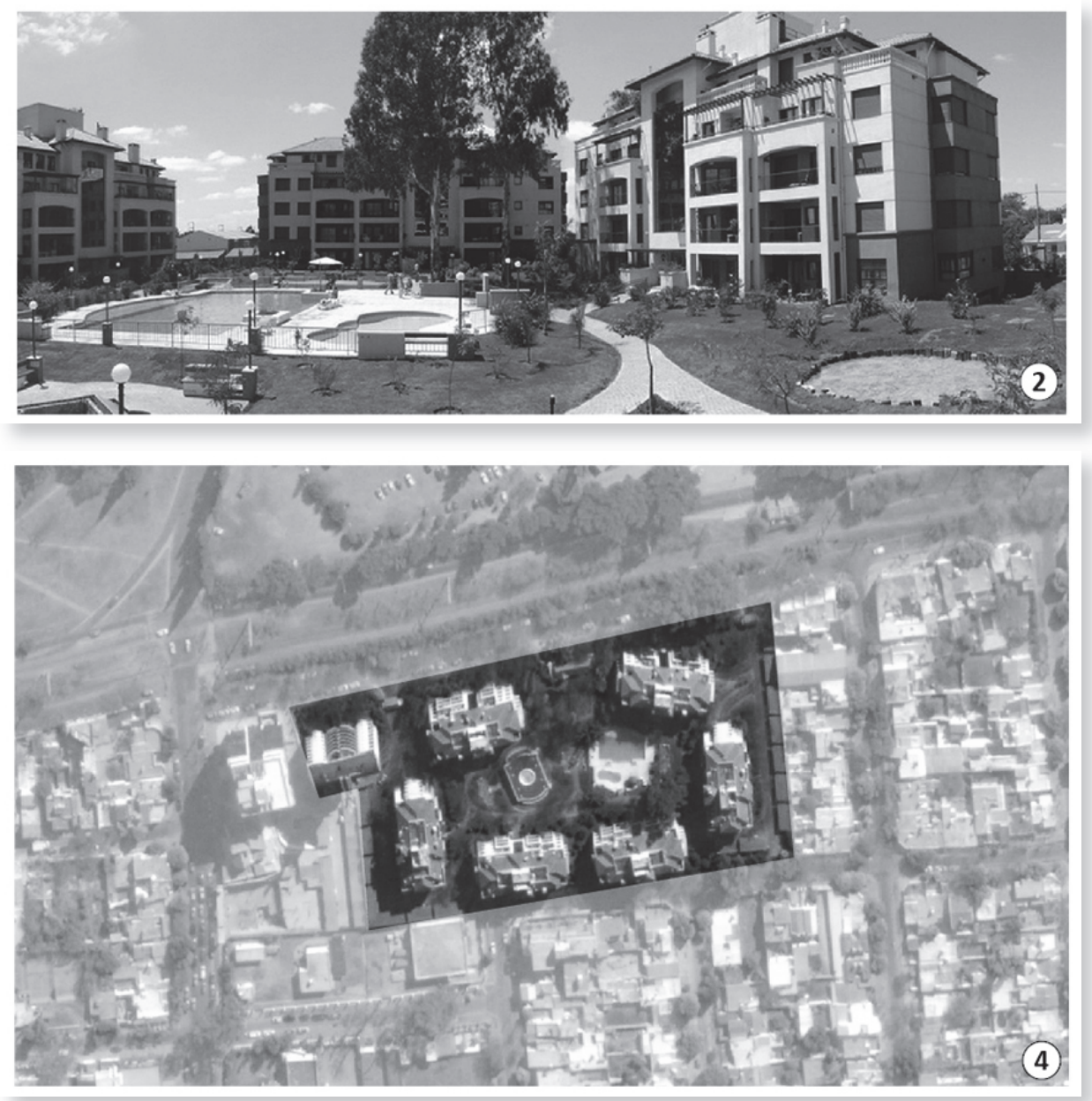

Figura 1. Planimetría e imagen de URE Lomas de los Carolinos (U2) y de Casonas del Sur (C2). Elaboración propia. Fuentes: (1) fotografía de Martín Lemma; (2) www.revistacalamo.com.ar, (3) y (4) Google Earth. 


\section{Cecilia Marengo y Martín Lemma}

4. La Ordenanza $8606 / 91$ regula el desarrollo de las URE. Así mismo, las ordenanzas que regulan el fraccionamiento y la ocupación del suelo en el ejido municipal son la 8060/85 y la 8256/85, respectivamente.

5. Como lo exigen las normas de fraccionamiento y ocupación del suelo, que, sancionadas en 1985 habían logrado poner freno a la expansión de loteos sin infraestructura (producido

durante la etapa de industrialización) y a la materialización de conjuntos de vivienda social en zonas de uso rural, pudiendo contener

(en ese momento) $e$ crecimiento dentro del perímetro definido como urbanizable.
Las urbanizaciones residenciales especiales (URE, por su encuadre normativo) se expanden a partir de 1991 en predios con cualidades ambientales y paisajísticas de interés en la periferia urbana, sobre corredores viales que posibilitan una rápida accesibilidad urbano-metropolitana. Las principales diferencias respecto de las ordenanzas que regulan el fraccionamiento y la ocupación del suelo en el ejido urbano, respectivamente, están dadas por el tamaño de espacio verde exigido (30 \% de la superficie del predio, cuando en los fraccionamientos tradicionales lo exigido es el $10 \%$ para área verde y el $5 \%$ para localización de equipamiento); la flexibilización de los requerimientos de localización, dado que la única restricción normativa es la existencia de usos peligrosos o incompatibles con el uso residencial, pudiendo localizarse en cualquier punto del ejido urbano mientras no interfieran en el sistema vial principal y, finalmente, las dimensiones mínimas de los lotes $\left(1000 \mathrm{~m}^{2}\right)$ exigidos. ${ }^{4}$ Inicialmente la normativa preveía condiciones de escala para la localización de las URE (hasta cincuenta lotes dentro de la avenida de circunvalación y más de cincuenta lotes fuera de esta), pero esta condición luego fue flexibilizada en 2004, según la Ordenanza 10760.

Como se puede observar en la figura 2, inicialmente se localizan en el eje de expansión noroeste y en la zona sur-sur oeste de la ciudad; en ambos casos- pero sobre todo en el noroeste - muy cercanos a barrios de altos ingresos. Ya en el período 2001-2010, solo se localizan en la zona sur (a excepción de un solo caso que se ubica en el límite del ejido municipal, en la ruta que conecta Córdoba con Carlos Paz). Cabe destacar que la gran mayoría de ellos está por fuera del anillo de circunvalación de la ciudad.

Las URE ofrecen acceso controlado, calles y seguridad privada, espacios verdes y de recreación y deportes para uso exclusivo de los habitantes y un entorno social homogéneo. Constituyen un tipo de urbanización radicalmente diferente en términos morfológicos del barrio tradicional cordobés, donde la expansión se producía por el trazado de la grilla abierta (amanzanamiento), con previsión de espacios verdes de carácter público (plaza) y de otros espacios exigidos para la localización futura del equipamiento comunitario (ver figura 3). ${ }^{5}$ El rasgo más significativo respecto de la urbanización tradicional abierta es el tamaño mínimo de los lotes exigidos, ya que normativamente se establece una superficie entre 1000 a $1500 \mathrm{~m}^{2}$ cuando los emprendimientos se localizan dentro de la avenida de circunvalación y entre 1500 a $2500 \mathrm{~m}^{2}$ de superficie, si están ubicados fuera de este límite. La dimensión de la parcela es la condición más significativa en términos de segmentación 
de la demanda, si consideramos que el lote medio en la ciudad de Córdoba tiene una superficie media de $250 \mathrm{~m}^{2}$ (MARENGO, 2008).

Por otra parte, a partir de 2001, el mercado inmobiliario desarrolla el modelo "casonas" que, a diferencia de las URE, no responde a una ordenanza única, sino a las posibilidades que ofrecen diferentes ordenanzas como las de áreas especiales o de redistribución de superficies, entre otras. ${ }^{6}$ Esta tipología constituye un caso singular, dado que surge a partir de 2001 y se replica con éxito durante la década incluyendo no solo los desarrollos de la empresa que inicia la propuesta, sino otras propuestas de características formales muy similares llevadas adelante por otras empresas desarrollistas. A los fines prácticos de este artículo, llamaremos "casonas" a todo este universo de casos, independientemente de la empresa que lo comercializa.

Las casonas se presentan en el mercado inmobiliario como departamentos con "estilo, distinción y elegancia”, "un estilo que combina la comodidad de una casa con los servicios y la seguridad de un departamento en condominios privados de baja altura, sumando amenities de jerarquía y espacios parquizados"7; en cercanías del centro o en áreas con centralidad y ventajas en términos de localización por la proximidad a corredores de servicio o barrios consolidados habitados por población de altos ingresos.

Como se verifica en la figura 2, la gran mayoría de las casonas, a diferencia de las URE, están localizadas dentro del anillo de circunvalación y, aun cuando no lo estén, se ubican en entornos consolidados y, por lo general, con buenos servicios urbanos. La tendencia de cambios de localización que se puede observar en la primera mitad de la década actual es la incorporación de lotes más alejados del centro, en particular hacia el suroeste de la ciudad (caso de la urbanización Manantiales).

De esta forma, se instala un modelo de uso y organización residencial de acceso controlado y seguridad privada, con características proyectuales semejantes en términos de altura de edificios (hasta cinco plantas); integrados por varios bloques de perímetro libre (de dos a ocho según los casos y dimensiones del predio) organizados en torno de un espacio central accesible a todos los residentes. Los complejos cuentan con espacios verdes de uso colectivo (para el conjunto) y oferta de servicios comunes (que incluyen pileta, gimnasio, salón de uso múltiple, entre otros).
6. La ordenanza de regulación de Áreas Especiales es la 10006/98 y sus ordenanzas modificatorias 10610/03, 10998/05 y 11400/07. La ordenanza que posibilita la de redistribución de superficies y nuevas alturas es la 11272/07.

7. Extractado de la página de la empresa desarrollista, www. edisur.com.ar. 

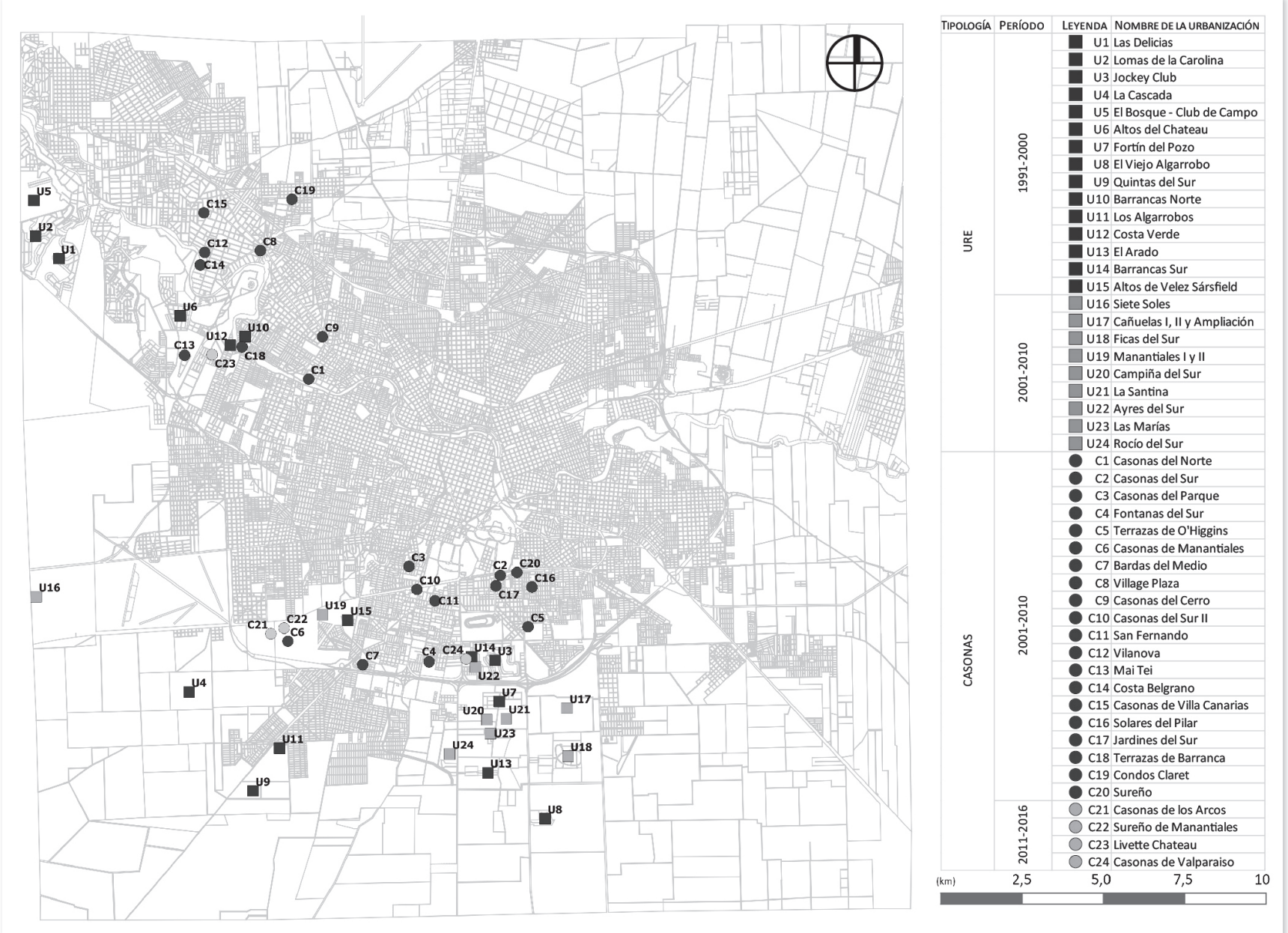

Figura 2. Localización de URE y casonas por período y nombre de comercialización. Elaboración propia Fuentes: Dirección de Planeamiento de la Municipalidad de Córdoba (para las URE) y Google Earth (para las casonas) 


\section{Formas de los emprendimientos}

La figura 3, que muestra comparativamente los perímetros y superficies a escala en los dos tipos de urbanizaciones antes mencionadas, permite observar la evolución de las formas urbano-arquitectónicas hacia emprendimientos de menor escala.

Para el caso de las URE, se puede observar que las formas irregulares de sus perímetros responden a las representaciones del parcelamiento rural, posteriormente incorporado como área urbanizable. La comparación a escala de las tipologías da cuenta de la gran variación en términos de superficies entre los emprendimientos, como de la diversidad proyectual adoptada en el diseño urbanístico (ver figura 4).

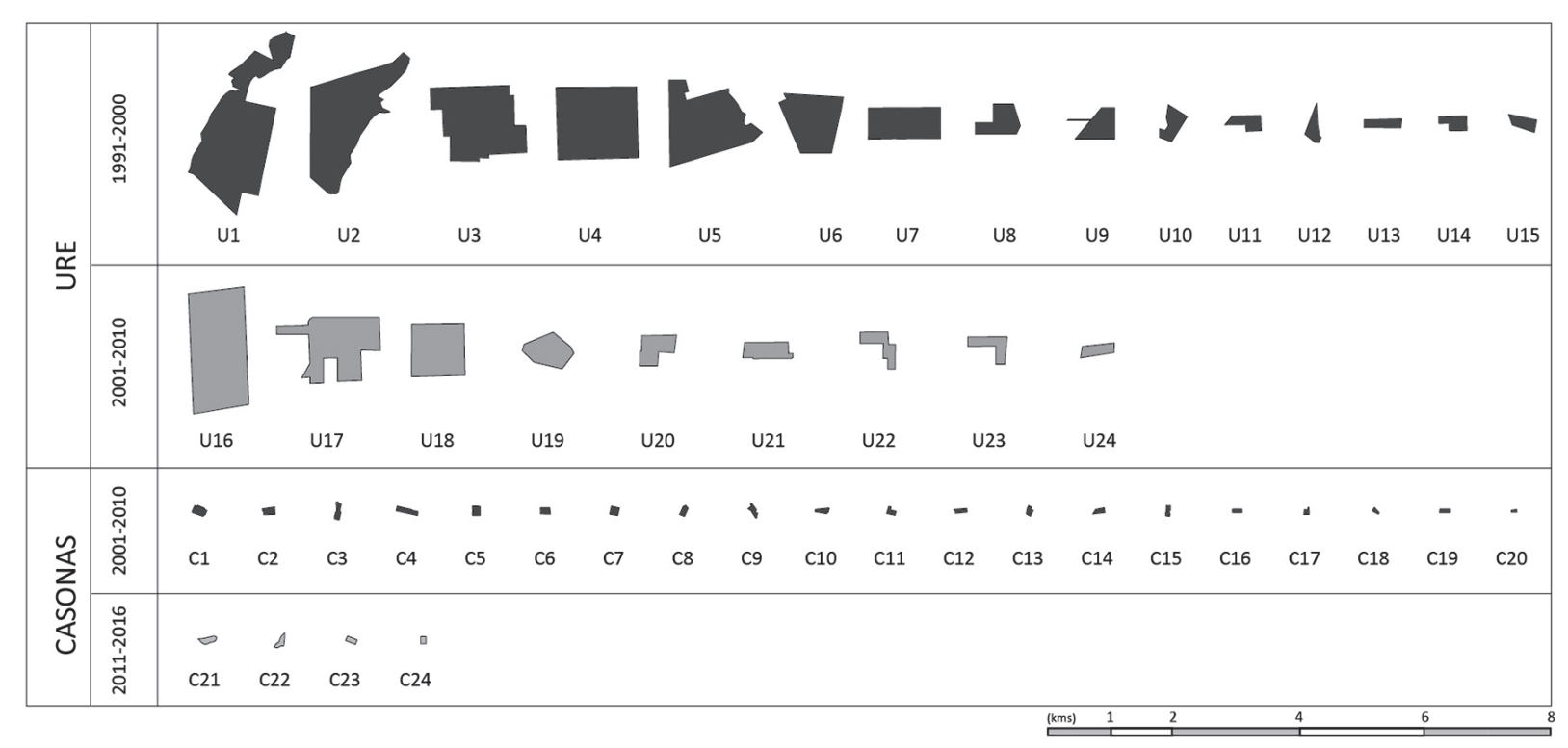

Figura 3. Evolución de la escala y forma de URE y casonas por período (ordenadas por superficie). Elaboración propia Fuentes: Dirección de Planeamiento de la Municipalidad de Córdoba y Google Earth 


\begin{tabular}{|c|c|c|c|c|c|}
\hline & 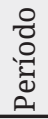 & $\begin{array}{l}\mathrm{N} .{ }^{\circ} \\
\text { ID }\end{array}$ & $\begin{array}{l}\text { Nombre de la } \\
\text { urbanización }\end{array}$ & $\begin{array}{l}\text { Superficie } \\
\text { Predio }\left(\mathrm{m}^{2}\right)\end{array}$ & $\begin{array}{c}\text { Unidades } \\
\text { Residenciales }\end{array}$ \\
\hline \multirow{26}{*}{ 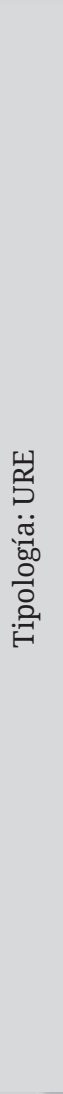 } & \multirow{15}{*}{ 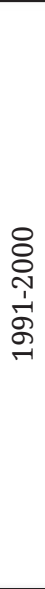 } & $\mathrm{U} 1$ & Las Delicias & 1.726 .251 & 412 \\
\hline & & $\mathrm{U} 2$ & Lomas de la Carolina & 1.460 .721 & 474 \\
\hline & & U3 & Jockey Club & 1.114 .665 & 402 \\
\hline & & $\mathrm{U} 4$ & La Cascada & 1.071 .292 & 412 \\
\hline & & U5 & El Bosque - Club de Campo & 988.908 & 397 \\
\hline & & U6 & Altos del Chateau & 555.701 & 252 \\
\hline & & U7 & Fortín del Pozo & 485.713 & 112 \\
\hline & & U8 & El Viejo Algarrobo & 185.655 & 78 \\
\hline & & U9 & Quintas del Sur & 175.747 & 69 \\
\hline & & U10 & Barrancas Norte & 121.440 & 59 \\
\hline & & U11 & Los Algarrobos & 79.026 & 35 \\
\hline & & U12 & Costa Verde & 75.272 & 37 \\
\hline & & U13 & El Arado & 74.051 & 33 \\
\hline & & U14 & Barrancas Sur & 73.211 & 45 \\
\hline & & U15 & Altos de Velez Sársfield & 59.122 & 30 \\
\hline & & & $\begin{array}{l}\text { Subtotal (sup., unid., dens.) } \\
\text { y Promedio (tiempos) }\end{array}$ & 8.246 .775 & 2847 \\
\hline & \multirow{10}{*}{ 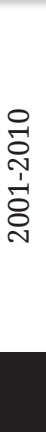 } & U16 & Siete Soles & 1.434 .771 & 575 \\
\hline & & U17 & Cañuelas I, II y Ampliación & 912.596 & 382 \\
\hline & & U18 & Ficas del Sur & 589.917 & 198 \\
\hline & & U19 & Manantiales I y II & 262.152 & 153 \\
\hline & & $\mathrm{U} 20$ & Campiña del Sur & 178.899 & 76 \\
\hline & & U21 & La Santina & 156.387 & 71 \\
\hline & & $\mathrm{U} 22$ & Ayres del Sur & 132.253 & 89 \\
\hline & & U23 & Las Marías & 123.615 & 49 \\
\hline & & $\mathrm{U} 24$ & Rocío del Sur & 59.839 & 23 \\
\hline & & & $\begin{array}{l}\text { Subtotal (sup., unid., dens.) } \\
\text { y Promedio (tiempos) }\end{array}$ & 3.850 .428 & 1616 \\
\hline
\end{tabular}




\begin{tabular}{|c|c|c|c|c|c|}
\hline & $\begin{array}{l}0 \\
0 \\
0 \\
0 \\
0 \\
0\end{array}$ & $\begin{array}{l}\mathrm{N} .^{\circ} \\
\text { ID }\end{array}$ & $\begin{array}{l}\text { Nombre de la } \\
\text { urbanización }\end{array}$ & $\begin{array}{l}\text { Superficie } \\
\text { Predio }\left(\mathrm{m}^{2}\right)\end{array}$ & $\begin{array}{c}\text { Unidades } \\
\text { Residenciales }\end{array}$ \\
\hline \multirow{26}{*}{ 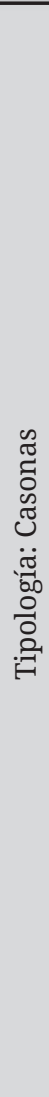 } & \multirow{20}{*}{ 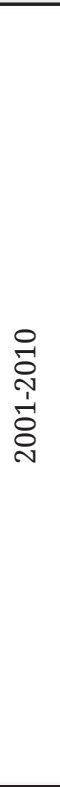 } & $\mathrm{C} 1$ & Casonas del Norte & 23.226 & 216 \\
\hline & & $\mathrm{C} 2$ & Casonas del Sur & 17.935 & 288 \\
\hline & & C3 & Casonas del Parque & 16.827 & 96 \\
\hline & & C4 & Fontanas del Sur & 16.156 & 160 \\
\hline & & $\mathrm{C} 5$ & Terrazas de O’Higgins & 15.430 & 118 \\
\hline & & C6 & Casonas de Manantiales & 13.671 & 120 \\
\hline & & C7 & Bardas del Medio & 13.610 & 96 \\
\hline & & $\mathrm{C} 8$ & Village Plaza & 11.593 & 59 \\
\hline & & C9 & Casonas del Cerro & 11.356 & 55 \\
\hline & & $\mathrm{C} 10$ & Casonas del Sur II & 10.757 & 120 \\
\hline & & C11 & San Fernando & 9.720 & 112 \\
\hline & & $\mathrm{C} 12$ & Vilanova & 9.439 & 48 \\
\hline & & $\mathrm{C} 13$ & Mai Tei & 9.109 & 87 \\
\hline & & C14 & Costa Belgrano & 8.826 & 44 \\
\hline & & C15 & Casonas de Villa Canarias & 8.794 & 36 \\
\hline & & $\mathrm{C} 16$ & Solares del Pilar & 7.051 & 59 \\
\hline & & C17 & Jardines del Sur & 5.357 & 48 \\
\hline & & C18 & Terrazas de Barranca & 4.481 & 14 \\
\hline & & C19 & Condos Claret & 3.379 & 29 \\
\hline & & $\mathrm{C} 20$ & Sureño & 2.443 & 24 \\
\hline & \multirow{5}{*}{ 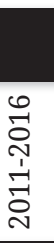 } & & $\begin{array}{l}\text { Subtotal (sup., unid., dens.) } \\
\text { y Promedio (tiempos) }\end{array}$ & 219.160 & 1829 \\
\hline & & $\mathrm{C} 21$ & Casonas de los Arcos & 18.687 & 144 \\
\hline & & $\mathrm{C} 22$ & Sureño de Manantiales & 14.229 & 80 \\
\hline & & $\mathrm{C} 23$ & Livette Chateau & 10.016 & 90 \\
\hline & & $\mathrm{C} 24$ & Casonas de Valparaiso & 7.374 & 48 \\
\hline & & & $\begin{array}{l}\text { Subtotal (sup., unid., dens.) } \\
\text { y Promedio (tiempos) }\end{array}$ & 50.306 & 362 \\
\hline
\end{tabular}



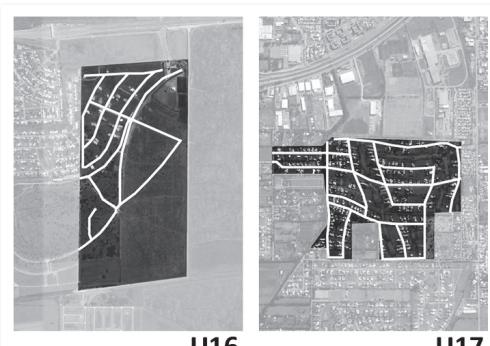

U17

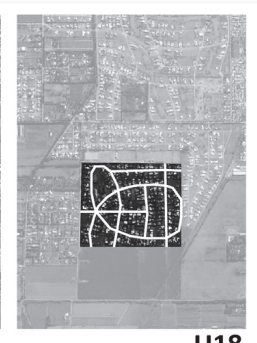

U18

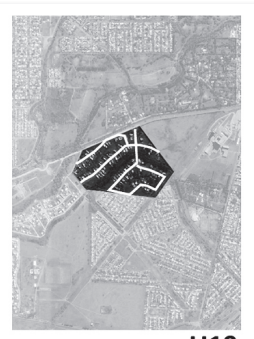

U19

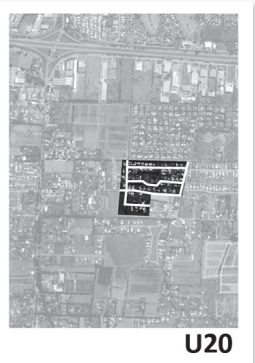

U20

Figura 4. Trazados de las cinco URE de mayor superficie (2001-2010). Elaboración propia Fuente: Google Earth

\section{Superficies y unidades residenciales}

En el período 1991-2000 se desarrollan quince URE que anexan una superficie urbanizada de 824,6 hectáreas. En el período 2001-2010, continúa esta tendencia, aunque con una menor cantidad de urbanizaciones y de menor tamaño. Se agregan nueve emprendimientos más, que suman 385 hectáreas. Este valor representa una reducción del $67 \%$ en cantidad de suelo utilizado por la misma tipología en una franja temporal equivalente. En total y considerando ambas décadas se incorporan 1209,6 hectáreas y 4463 lotes y se estima igual cantidad de unidades de vivienda (tabla 1).

En el caso de las URE se observa que las de mayor escala se materializan en el período 1991-2000, y se registran al menos cinco urbanizaciones que superan los 397 lotes. En el período 2001-2010, se incorporan 1616 lotes (unidades residenciales). Del análisis se desprende que se reduce considerablemente la escala de las intervenciones, ya que se registran solo dos conjuntos integrados por 382 y 575 lotes, lo cual estaría señalando la saturación de este tipo de oferta (figura 3).

En el período 2001-2010 se construyen veinte emprendimientos tipo casonas, que abarcan una superficie de 21,1 hectáreas y un total de 1829 unidades de vivienda. Este valor -comparativamente con los 1616 lotes relevados en el caso de las URE- señala la aceptación por parte de la demanda de este tipo de modo de habitar, asociado a propuestas organizativas que prevén una mayor compacidad en el tejido, bajo el régimen de vivienda colectiva. 
En 2016, continúa el desarrollo de estas propuestas, ya que se registran cuatro nuevos emprendimientos que suman a la oferta 362 unidades residenciales (tabla 2). Se puede observar que aun cuando recién ha transcurrido la mitad de la década, existiría una tendencia al decrecimiento en la cantidad de emprendimientos y unidades residenciales construidas, lo cual podría estar indicando una saturación de este tipo de oferta en el mercado. Si bien las casonas presentan variación de tamaño entre los diferentes emprendimientos, estas no son tan significativas como en el caso de las URE.

\section{Densidad}

Las dos formas urbanas residenciales presentan diferencias significativas en términos de densidad. Dado que las propuestas son disímiles, se consideró como medida la densidad bruta (viviendas por hectáreas) a los fines de aportar datos comparativos.

En las URE el valor más alto de densidad asciende a 6,7 viv./ha y el mínimo, a 2,3 viv./ha. El valor medio en 1991-2001 es de 3,5 viv./ha y en 2001-2010, de 4,2 viv./ha, lo cual demuestra un aumento relativo de la densidad bruta (del $20 \%$ ). Si bien se trata de densidades brutas, los valores indican el alto grado de dispersión y la baja compacidad de esta forma urbana, en una forma de ocupación claramente insustentable en términos de consumo de suelo por habitante.

En las casonas, la densidad bruta alcanza valores medios de 83,5 viv./ha en el período 2001-2010. En promedio son complejos casi veinte veces más densos que las URE en el mismo período. Los valores máximos y mínimos oscilan entre las 160 viv./ha y las 31,2 viv./ha, con un modelo de ocupación más compacto, eficiente y sustentable en términos de consumo de suelo por habitantes. A su vez, la densidad bruta promedio para el período 2001-2010 es mayor que la del período anterior para el caso de las URE y que la del período posterior para el caso de las casonas (tablas 1 y 2).

Por otra parte, si analizamos la localización de los emprendimientos con los valores de densidad (cruzamos los datos de la tabla 1 y tabla 2 con la figura 2), observamos que las seis URE más densas (U22, U14, U19, U15, U12 y U10) están localizadas dentro del anillo de circunvalación, tal como lo preveía la Ord. 8606/91. La única que no cumple con esta condición de densidad es U3 - Jockey Club. 


\section{Cecilia Marengo y Martín Lemma}

En las casonas, se puede observar que las seis menos densas (C18, C15, C9, C14, C12 y C8) están localizadas en el corredor noroeste (eje preferencial de crecimiento), aun cuando en las Bases del Plan Director 2020 se preveía un programa de densificación para este corredor. Cabe aclarar que hay solo un emprendimiento que no es de baja densidad localizado en este corredor (C19 - Condos Claret).

\section{Accesibilidad}

Se indagó sobre las condiciones de accesibilidad y movilidad que median entre la distancia que presentan estas dos formas de ocupación. En las tablas 1 y 2 se muestran los tiempos de desplazamiento desde las puertas principales de las urbanizaciones hasta un punto céntrico de fácil acceso determinado por el cruce de la avenida Vélez Sarsfield y el bulevar San Juan. Fueron tenidos en cuenta dos medios de transporte: automóvil particular y transporte urbano de pasajeros (TUP). Cabe aclarar que hay tres URE que no cuentan con TUP, pero sí con otros servicios metropolitanos cuyos datos de frecuencias y tiempos no son de libre acceso.

Para las URE, se observa que el tiempo promedio para llegar al centro de la ciudad es de 20 minutos en auto (con tiempos entre 16 y 25 minutos) y de 63 minutos en TUP (con tiempos entre 44 y 77 minutos). Para las casonas, el tiempo es de 20 minutos en auto (con valores entre 9 y 30 minutos) y de 48 minutos en TUP (con valores entre 27 y 66 minutos). Estos datos demuestran que para las URE el tiempo al centro en auto es 3,15 veces menor que en TUP y solo 2,4 veces menor para las casonas. En segundo lugar, se observa que los tiempos medios de desplazamiento en auto son similares, aun cuando las casonas tienen, en términos generales, localizaciones más próximas al área central. En esta ecuación inciden fuertemente la mayor amplitud de valores que se registra para las casonas y las modificaciones en la movilidad urbana que han mejorado las condiciones de accesibilidad de las URE.

Si consideramos el TUP, observamos que el tiempo promedio de desplazamiento para las casonas es un 75 \% menor comparado con las URE, en lo cual inciden las condiciones de localización y el mayor desarrollo de la red de transporte urbano. Finalmente, observamos que el tiempo en TUP para casonas con mejor accesibilidad y cercanía al centro es similar al tiempo en auto para las URE con localizaciones menos favorables. Es decir que podríamos confirmar lo que señala InDovina (2004), que los patrones de densidad e intensidad de usos que 
caracterizan a la ciudad consolidada podrían ser equivalentes a los de accesibilidad/movilidad de la ciudad difusa, dependiendo del modo de transporte.

\begin{tabular}{|c|c|c|c|c|c|c|c|c|}
\hline \multicolumn{2}{|c|}{ Tabla I } & \multicolumn{7}{|c|}{ URE (1991-2010) } \\
\hline & $\begin{array}{l}0 \\
8 \\
0 \\
0\end{array}$ & $\begin{array}{l}\mathrm{N} .^{\circ} \\
\text { ID }\end{array}$ & $\begin{array}{l}\text { Nombre de la } \\
\text { urbanización }\end{array}$ & $\begin{array}{l}\text { Superficie } \\
\text { Predio }\left(\mathrm{m}^{2}\right)\end{array}$ & $\begin{array}{c}\text { Unidades } \\
\text { Residenciales }\end{array}$ & $\begin{array}{c}\text { Densidad } \\
\text { Bruta } \\
\text { (viv/ha) }\end{array}$ & $\begin{array}{l}\text { Tiempo al } \\
\text { centro en } \\
\text { auto (mín.) }\end{array}$ & $\begin{array}{l}\text { Tiempo al } \\
\text { centro en } \\
\text { TUP (mín.) }\end{array}$ \\
\hline \multirow{26}{*}{ 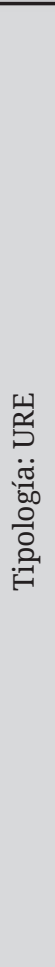 } & \multirow{15}{*}{ 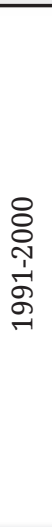 } & U1 & Las Delicias & 1.726 .251 & 412 & 2,4 & 28 & - \\
\hline & & $\mathrm{U} 2$ & Lomas de la Carolina & 1.460 .721 & 474 & 3,2 & 29 & - \\
\hline & & $\mathrm{U} 3$ & Jockey Club & 1.114 .665 & 402 & 3,6 & 15 & 49 \\
\hline & & $\mathrm{U} 4$ & La Cascada & 1.071 .292 & 412 & 3,8 & 23 & 75 \\
\hline & & U5 & El Bosque - Club de Campo & 988.908 & 397 & 4,0 & 32 & 104 \\
\hline & & U6 & Altos del Chateau & 555.701 & 252 & 4,5 & 28 & 66 \\
\hline & & U7 & Fortín del Pozo & 485.713 & 112 & 2,3 & 21 & 64 \\
\hline & & U8 & El Viejo Algarrobo & 185.655 & 78 & 4,2 & 23 & 74 \\
\hline & & U9 & Quintas del Sur & 175.747 & 69 & 3,9 & 24 & 57 \\
\hline & & U10 & Barrancas Norte & 121.440 & 59 & 4,9 & 24 & 48 \\
\hline & & U11 & Los Algarrobos & 79.026 & 35 & 4,4 & 23 & 47 \\
\hline & & U12 & Costa Verde & 75.272 & 37 & 4,9 & 26 & 60 \\
\hline & & U13 & El Arado & 74.051 & 33 & 4,5 & 22 & 78 \\
\hline & & U14 & Barrancas Sur & 73.211 & 45 & 6,1 & 17 & 44 \\
\hline & & U15 & Altos de Velez Sársfield & 59.122 & 30 & 5,1 & 14 & 41 \\
\hline & & & $\begin{array}{l}\text { Subtotal (sup., unid., dens.) } \\
\text { y Promedio (tiempos) }\end{array}$ & 8.246.775 & 2847 & 3,5 & 23 & 62 \\
\hline & \multirow{9}{*}{ 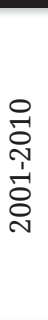 } & U16 & Siete Soles & 1.434 .771 & 575 & 4,0 & 25 & - \\
\hline & & U17 & Cañuelas I, II y Ampliación & 912.596 & 382 & 4,2 & 21 & 74 \\
\hline & & U18 & Ficas del Sur & 589.917 & 198 & 3,4 & 22 & 77 \\
\hline & & U19 & Manantiales I y II & 262.152 & 153 & 5,8 & 16 & 51 \\
\hline & & $\mathrm{U} 20$ & Campiña del Sur & 178.899 & 76 & 4,2 & 19 & 59 \\
\hline & & $\mathrm{U} 21$ & La Santina & 156.387 & 71 & 4,5 & 19 & 66 \\
\hline & & U22 & Ayres del Sur & 132.253 & 89 & 6,7 & 17 & 44 \\
\hline & & $\mathrm{U} 23$ & Las Marías & 123.615 & 49 & 4,0 & 20 & 67 \\
\hline & & $\mathrm{U} 24$ & Rocío del Sur & 59.839 & 23 & 3,8 & 19 & 68 \\
\hline & & & $\begin{array}{l}\text { Subtotal (sup., unid., dens.) } \\
\text { y Promedio (tiempos) }\end{array}$ & 3.850 .428 & 1616 & 4,2 & 20 & 63 \\
\hline
\end{tabular}

Fuentes: Dirección de Planeamiento de la Municipalidad de Córdoba (superficies de predios y unidades residenciales), Google Maps (tiempos de desplazamiento al centro) y elaboración propia (densidad bruta) 
Tabla II Casonas (2001-2016)

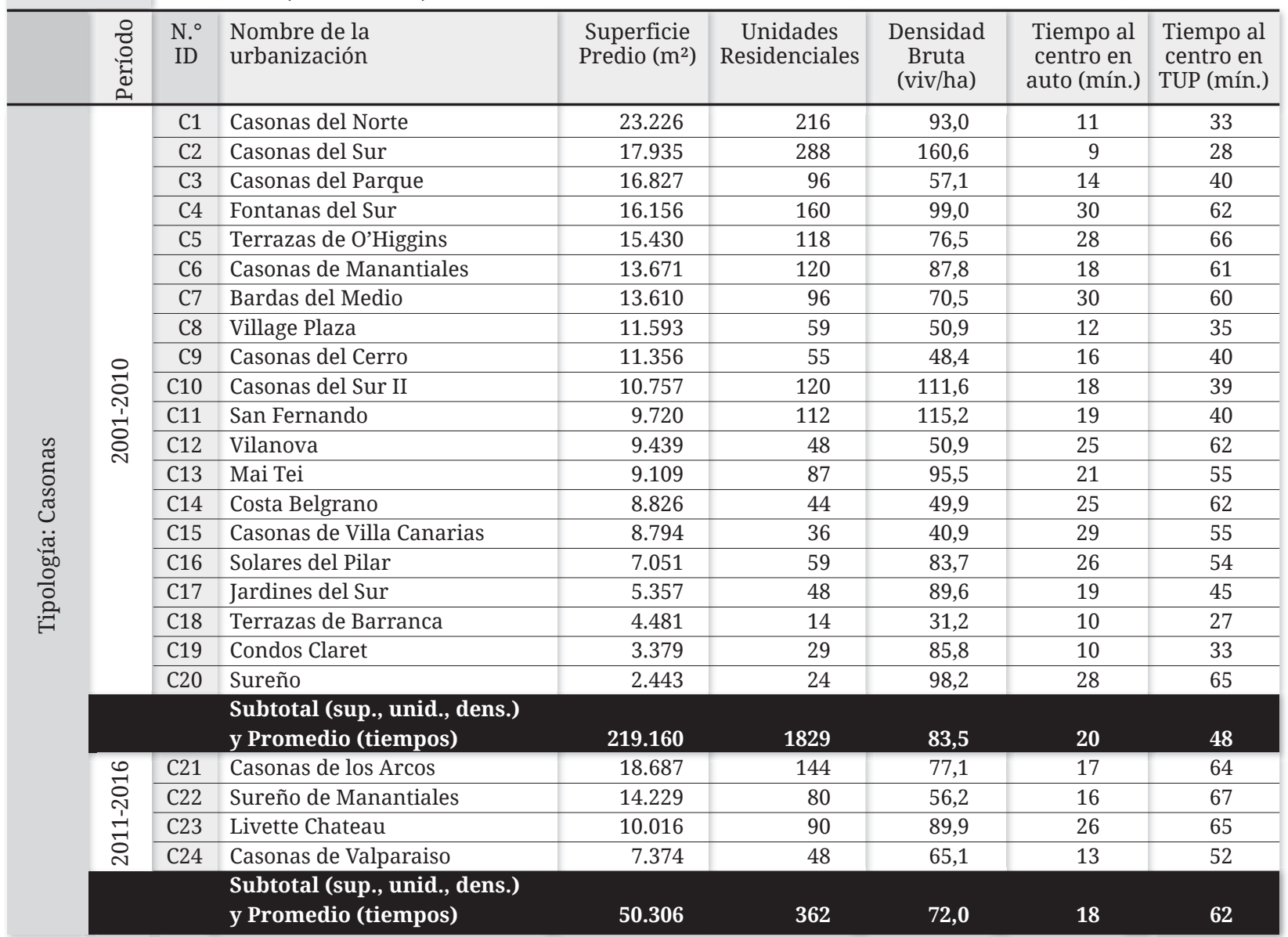

Fuentes: Dirección de Planeamiento de la Municipalidad de Córdoba (superficies de predios y unidades residenciales), Google Maps (tiempos de desplazamiento al centro) y elaboración propia (densidad bruta) 


\section{Reflexiones finales}

$\mathrm{Al}$ examinar las formas construidas en los emprendimientos residenciales promovidos por el mercado inmobiliario en la ciudad de Córdoba en el período de análisis, comprobamos que responden a dos dinámicas urbanísticas opuestas en términos de crecimiento urbano: nuevas extensiones periféricas (URE) y re-densificación/renovación en áreas urbanizadas (casonas). El amplio desarrollo de ambos tipos de propuestas en el caso de Córdoba da cuenta de los niveles de inversión inmobiliaria presentes en la ciudad durante la década analizada; la superficie urbanizada, la cantidad de lotes y unidades de viviendas señalan, como menciona De MATTos (2010), el impacto de la gestión empresarial en el desarrollo urbano residencial.

Ambos modelos de crecimiento, tanto los que se asocian con una mayor dispersión de la forma urbana - resultado de la descentralización de funciones y las mejoras en la accesibilidad urbano/metropolitana - como los que se materializan en las áreas sujetas a procesos de renovación, presentan nuevas formas residenciales que tienen en común erigirse como fragmentos (con cerramiento perimetral) destinados a población de altos ingresos. Las casonas, en tanto ocupación más compacta, representan condiciones de mayor sustentabilidad y eficiencia en el uso del suelo y provisión de servicios, aunque al igual que las URES conforman enclaves socialmente homogéneos y físicamente fragmentados.

El análisis empírico permite concluir que el concepto de ciudad difusa que propone INDOVINA ofrece una nueva interpretación de las formas urbanas en la ciudad y es válido para explicar las preferencias por un tipo de urbanización de localización periférica, donde el acento está puesto en las condiciones de accesibilidad (en tiempos de desplazamiento) bajo condiciones caracterizadas por la movilidad individual generalizada en auto, por sobre las condiciones de densidad poblacional e intensidad de usos, propias de la urbanización compacta. La caracterización de los tipos urbano-residenciales y su evolución en el período presenta condiciones disímiles en términos de sustentabilidad. El consumo de suelo, la densidad edilicia y la accesibilidad representan las variables de intervención más significativas para valorar los efectos de este tipo de crecimiento en el sistema urbano y repensar, a la luz de las formas residenciales desarrolladas por el mercado, nuevos modelos explicativos de la urbanización en el siglo XXI. 


\section{Bibliografía}

CASTELLS, Manuel (1995) La ciudad informacional: tecnologías de la información, reestructuración económica y el proceso urbano-regional. Alianza Editorial, Madrid.

DE MATTOS, Carlos A. (2001). “Metropolización y suburbanización”. En: Eure, 27 (80), 5-8. (2010). "Una nueva geografía latinoamericana en el tránsito de la planificación a la gobernanza, del desarrollo al crecimiento”. En: Eure, 36 (108), 167-179. DE SOLÀ-MORALES RUBIÓ, Manuel (1973) Las formas de crecimiento urbano. Programa "Urbanística I". Escola Tècnica Superior d’Arquitectura de Barcelona, Barcelona. INDOVINA, Francesco (2004). "La Ciudad Difusa”. En: A. M. Ramos (Ed.) Lo urbano en 20 autores contemporáneos (pp. 19-43). Universidad Politècnica de Catalunya, Barcelona.

JENKS, Mike, KOZAK, Daniel y TAKKANON, Pattaranan (2008). "Introduction: world cities and urban form”. En: M. Jenks, D. Kozak, y P. Takkanon (Eds.) World cities and urban form. Fragmented, polycentric, sustainable? (pp. 3-8). Routledge, Nueva York.

MARCUSE, Peter y VAN KEMPEN, Ronald (2000) Globalizing Cities. A new spatial order? Blackwell Publishers, Oxford.

MARENGO, Cecilia (2008) Urban Sprawl and spatial planning: facing the challenges of growing social inequity. Case study: Córdoba, Argentina. TU Delft.

MOUDON, Anne (1997). "Urban morphology as an emerging interdisciplinary field". En: Urban Morphology, 1, 3-10.

NEUMAN, Michael (2005). “The Compact City Fallacy”. En: Journal of Planning Education and Research, 25 (1), 11-26.

SASSEN, Saskia (Ed.) (2002) Global networks Linked cities. Routledge, New York. 